\title{
Los estudios de Grado en Información y Documentación en España. De los antecedentes a la situación actual: una visión crítica
}

\author{
Degree studies in Information and Documentation in Spain. \\ From their antecedents to the current situation: A critical view
}

\author{
Antonio MUÑOZ-CAÑAVATE1 (iD 0000-0003-2032-6916 \\ Verónica LARIOS-SUÁREZ² (D) 0000-0002-7699-8968
}

\begin{abstract}
Resumen
Los estudios universitarios en Documentación se remontan en España a los años ochenta del siglo XX cuando comienza a impartirse la Diplomatura en Biblioteconomía en Documentación, a la que seguirán posteriormente los estudios de Licenciado en Documentación y los Doctorados en esta especialidad. El proceso de adaptación de la Universidad española al Espacio Europeo de Educación Superior tiene como resultado la aparición de los estudios universitarios de Grado en Información y Documentación. Este trabajo presenta el origen de estos estudios de Grado, su desarrollo y situación actual, pero también sus antecedentes, sin los cuales no es posible entender la realidad actual. Se analiza la denominada "crisis de los estudios de Documentación en España" y la necesidad de repensar los estudios universitarios en este país.
\end{abstract}

Palabras clave: Biblioteconomía. Documentación. España. Universidad.

\begin{abstract}
University studies in Documentation Sciences in Spain go back to the 1980s with the implementation of the University degree in Librarianship and Documentation. This was followed later by the Licentiate degree (roughly equivalent to the M.A. degree) and Doctorate courses in Documentation. The process of adapting Spain's university system to the European Space for Higher Education resulted in the appearance of Bachelors' degree courses in Information and Documentation. This study presents the origin of these degree courses, their development and current situation, and their antecedents, without which, would be impossible to understand the current reality. The so-called "crisis of Documentation studies in Spain" is analyzed, together with the need to rethink the country's university studies.
\end{abstract}

Keywords: Librarianship. Documentation. Spain. University.

\section{Introducción}

La implantación de los estudios universitarios de Biblioteconomía y Documentación (ByD) en España tiene su origen en el último tercio del siglo XX, aunque como asignaturas aisladas ya estaban presentes en distintas titulaciones en España con anterioridad (Mateu-Ibars, 1981; Abadal, 1993).

\footnotetext{
1Universidad de Extremadura, Facultad de Ciencias de la Documentación y la Comunicación, Departamento de Información y Comunicación. Plazuela Ibw Marwan, s/n., 06001, Badajoz. España. Correspondencia para/Correspondence to: A. MUÑOZ-CAÑAVATE. E-mail: <amuncan@unex.es>.

${ }^{2}$ Diputación Provincial de Badajoz, Archivo Provincial. Badajoz. España.

Recibido el 8 del julio del 2017 y aprobado el 25 del octubre del 2017.
}

Como citar este artículo/How to cite this article

Muñoz-Cañavate, A.; Larios-Suárez, V. Los estudios de Grado en Información y Documentación en España. De los antecedentes a la situación actual: una visión crítica. Transinformação, v.30, n.3, p.336-347, 2018. http://dx.doi.org/10.1590/2318-08892018000300006 
Anteriormente, la formación que capacitaba para trabajar en centros bibliotecarios y archivísticos se remonta en España a mediados del siglo XIX cuando se crea la Escuela de Diplomática que desaparecerá en 1900. Posteriormente, en 1915 se crea en Barcelona la Escuela de Bibliotecarias. En 1947 aparece la Escuela de Formación Técnica de Archivos, Bibliotecas y Museos, antecedente de la Escuela de Documentalistas de Madrid nacida en 1964, que en 1977 cambia su denominación a Escuela de Bibliotecarios, y en 1980 se convierte en Centro de Estudios Bibliográficos y Documentarios. Este centro desaparece definitivamente en 1986.

Sin embargo la formación en Biblioteconomía y Documentación que en la actualidad se imparte en la universidad española tiene su fecha de arranque en 1978 cuando se publica el Real Decreto 3104/1978 por el que se crean en la educación universitaria las enseñanzas de Biblioteconomía y Documentación, y sobre todo en febrero de 1981 cuando se publica la Orden Ministerial que desarrolla las directrices de los planes de estudio de Diplomado en Biblioteconomía en la Universidad española. Este proceso de creación y desarrollo de los estudios universitarios ha sido estudiado en España por diversos autores (Abadal, 1993; Abadal; Miralpeix, 1999; Fuentes; González, 2001; Chaín-Navarro; Muñoz-Cañavate, 2009a; Moneda-Corrochano, 2014). Aunque la Universidad española sufre un proceso de reformas a finales de los años ochenta, a través de la Ley Orgánica 11/1983 (España,1983) y el Real Decreto 1497/1987 (España, 1987) que da lugar al cambio de los planes de estudio de las Diplomaturas de ByD que ya se habían implantado en algunas universidades.

La aparición de los estudios de Licenciado en Documentación supuso una reivindicación desde la misma aparición de los estudios de Diplomado en Biblioteconomía y Documentación. Desde las primeras promociones de Diplomados en Biblioteconomía y Documentación se venía exigiendo la necesidad de ampliar la oferta formativa a un segundo ciclo. El objetivo del mismo era dar cabida en el mercado de trabajo a profesionales con un sesgo profesional distinto al de los diplomados. Es decir, se trataba de formar gestores y, a su vez, de abrir una vía de acceso a la investigación a través de los programas de Doctorado. En algunas universidades los estudios de tercer ciclo se adelantaron a los estudios de segundo ciclo. Los esfuerzos decisivos para la consecución final del objetivo propuesto vinieron dados por la Comisión Nacional de Directores y Responsables de Escuelas y Diplomaturas de Biblioteconomía y Documentación, y por el alumnado de toda España agrupado en torno a la Asociación de Diplomados y Alumnos en Biblioteconomía y Documentación (Adab).

En 1992 se publican finalmente las Directrices para la elaboración del Título de Licenciado en Documentación, aprobado por R.D. 912/1992 (España, 1992). Unos estudios universitarios de segundo ciclo con dos cursos académicos, a los que podían acceder los Diplomados en Biblioteconomía y Documentación, pero también los titulados con otros títulos universitarios que debían cursar unos complementos de formación. El título de Licenciado en Documentación debía capacitar para la dirección de los archivos, bibliotecas, y centros de documentación. En el curso 1994-1995 comenzó por primera vez la Licenciatura en Documentación en varias universidades.

La planificación de estos estudios de Diplomatura y de Licenciatura no estuvo exenta de controversia, ya que en algunas universidades que sólo ofrecían la Licenciatura, los planes de estudio eran muy similares a las universidades que sólo ofrecían la Diplomatura (Cuadro 1).

Además surgirán los estudios de Doctorado específicos en Documentación (Muñoz-Cañavate; Larios-Suárez, 2017). Posteriormente, el proceso de adaptación de todos los estudios universitarios al Espacio Europeo de Educación Superior (EEES) supondrá de facto la desaparición de las Diplomaturas y las Licenciaturas de Documentación y su sustitución por un nuevo título: el Grado de Información y Documentación. 
Cuadro 1. Año de inicio de las Diplomaturas y Licenciaturas.

\begin{tabular}{|c|c|c|}
\hline Universidad & $\begin{array}{c}\text { Diplomatura de Biblioteconomía y } \\
\text { Documentación (tres años) }\end{array}$ & Licenciatura en Documentación (dos años) \\
\hline León & 1991 & \\
\hline San Pablo CEU & 1994 & \\
\hline Valencia Estudi General & 1996 & \\
\hline Vic & 1998 & \\
\hline Zaragoza & 1989 & \\
\hline Barcelona & 1982 & 1998 \\
\hline Carlos III de Madrid & 1990 & 1994 \\
\hline Complutense de Madrid & 1990 & 1996 \\
\hline Extremadura & 1994 & 1997 \\
\hline Granada & 1983 & 1994 \\
\hline La Coruña & 1996 & 2003 \\
\hline Salamanca & 1987 & 1994 \\
\hline Universidad de Alcalá de Henares & & 1994 \\
\hline Universidad Autónoma de Barcelona & & 1999 \\
\hline Universitat Oberta de Catalunya & & 1999 \\
\hline Universidad Politécnica de Valencia & & 1997 \\
\hline
\end{tabular}

\section{La reforma universitaria: Llegada de la interdisciplinariedad a los estudios de Documentación}

Como se ha señalado antes, la Universidad española sufre una importante reforma a finales de los años ochenta del siglo XX (anterior a la que llegará después con el EEES). Este proceso de reformas supone para ambas titulaciones (Diplomatura y Licenciatura) tanto la llegada de profesorado de otras áreas universitarias de conocimiento, ajenas al área tradicional y mayoritaria de Biblioteconomía y Documentación que podían impartir clases en los títulos de Diplomatura en Biblioteconomía y Licenciado en Documentación, como la posibilidad de que el profesorado del área de conocimiento de Biblioteconomía y Documentación pueda impartir clases en otras titulaciones diferentes. Lo que había hecho la Ley de Reforma Universitaria, que da origen a todos estos cambios, fue asumir las demandas sociales y universitarias que exigían más flexibilidad de los títulos y de sus currícula con el objetivo de adaptar la formación universitaria a la realidad del mercado laboral. Hasta entonces el monopolio en la enseñanza de las titulaciones ejercido hasta el momento por determinadas áreas de conocimiento, será sustituido por una mayor interdisciplinariedad.

El estudio de Sanz-Casado et al. (2001) reflejaba la diversidad de áreas de conocimiento que se encontraban en las dos titulaciones. La necesidad de disponer de conocimientos en tecnologías, Derecho, Economía, Estadística o Ciencias y técnicas historiográficas obligó a una mayor permeabilidad de las titulaciones de Documentación, a las que accedieron áreas de conocimiento de muy distinta naturaleza. Este estudio realizaba una confrontación entre el peso del área de Biblioteconomía y Documentación (ByD) con otras áreas de conocimiento. En el conjunto de las Diplomaturas de Biblioteconomía, impartidas en las diferentes universidades, el área de ByD tenía un participación del $72,20 \%$ en el conjunto de las asignaturas, frente al $27,80 \%$ de otras áreas de conocimiento, mientras para la titulación de Documentación el peso del área de ByD era del 66,33\% frente al 33,67\% de otras áreas.

La reforma universitaria y el cambio posterior de los planes de estudio permitirá que se impartan asignaturas de Documentación como materia troncal u optativa en varios planes de estudio de otras titulaciones (Abadal, 1993). La Documentación como asignatura troncal aparece, aunque con el sesgo correspondiente a la aplicación a cada uno de los planes de estudio en las siguientes titulaciones: Licenciatura en Periodismo, Licenciatura en Comunicación Audiovisual, Licenciatura en Publicidad y Relaciones Públicas, Licenciatura en Traducción e Interpretación y Diplomatura 
en Gestión y Administración Pública. Si bien como asignatura obligatoria y optativa (propuestas por cada universidad como materia específica en sus titulaciones) también tiene presencia en titulaciones como Humanidades, Historia, Químicas, Enfermería, Odontología de determinadas universidades (Olvera-Lobo; García-Caro, 2000).

\section{La reforma universitaria: la llegada de la libre elección: una oportunidad perdida}

Un caso interesante será la del último tipo de asignaturas que trae la reforma de los planes de estudio, la libre elección o configuración. La libre elección o configuración era un tipo de materia distinta a la titulación que cursaba el alumno, es decir el alumno debía realizar hasta un 10\% de su currículum en materias que podía libremente escoger entre las impartidas por la propia Universidad o por otra Universidad.

Para el Área de conocimiento de Biblioteconomía y Documentación, la libre elección pudo haber sido una oportunidad única para extenderse de manera mayoritaria en todos los títulos, ya que el Consejo de Universidades aprobó unas recomendaciones (18 de diciembre de 1996), donde formuló una serie de indicaciones a las universidades sobre la organización de los planes de estudio conducentes a títulos universitarios oficiales (España, 1997). En la recomendación segunda, referida a materias de libre elección se decía:

\footnotetext{
se sugiere que en el conjunto de materias, seminarios y actividades académicas que constituyen el objeto de la libre elección, se fomente la inclusión, como ampliación de las ya existentes, de materias específicas concebidas a tal efecto (por ejemplo, Derecho para no juristas); materias instrumentales (Informática, Documentación etc.); por su carácter introductory básico, asignaturas del primer curso de otras carreras, y materias que integran los complementos de formación exigidos para acceder a las titulaciones de que se trate en cada caso (España, 1997, p.1895).
}

Se hacía aquí referencia expresa a la Documentación. Sin embargo, tal y como demuestra el estudio de Olvera-Lobo y García-Caro la implantación de esta asignatura impartida en titulaciones ajenas a la Diplomatura de Biblioteconomía y Documentación o a la Licenciatura en Documentación apenas tuvo acogida (Olvera-Lobo; García-Caro, 2000).

\section{Los estudios de Grado en Información y Documentación}

En 1999 como consecuencia de la Declaración de Bolonia, treinta países europeos sientan las bases para la construcción de un espacio universitario homogéneo en Europa. Surge así el Espacio Europeo de Educación Superior lo que supone un cambio en las normativas legales que rigen los títulos universitarios, además de la instauración de un proceso de acreditación de los títulos que tienen que ser verificados por un organismo estatal y realizar evaluaciones periódicas.

Los títulos universitarios se clasifican en estudios de Grado y en estudios de Postgrado. En las titulaciones de Documentación desaparecen, como se señaló antes, las antiguas Diplomaturas en Biblioteconomía y Documentación, y Licenciaturas en Documentación. Surge, así, el Grado de Información y Documentación, los Máster de distintos tipos y los Doctorados adaptados al EEES.

En 2016 el Gobierno español aprobó la equivalencia del título de Diplomado en Biblioteconomía y Documentación al nivel de Grado de los nuevos estudios del EEES (España, 2016a) y la equivalencia del título de Licenciado en Documentación al nivel de Máster del EEES (España, 2016b).

Es en el curso 2008-2009 cuando comienza la impartición en la Universidad española de los estudios de Grado de Información y Documentación adaptados al EEES. De las 17 universidades que impartían los estudios de 
Diplomatura y Licenciatura en Documentación - previos a la reforma del Espacio Europeo de Educación Superior - doce han continuado con los estudios de Grado en Información y Documentación, que sustituyen a la Diplomatura de Biblioteconomía y Documentación (Cuadro 2).

Cuadro 2. Grados de Información y Documentación.

\begin{tabular}{|c|c|c|c|}
\hline Universidad & Curso de inicio & Número de créditos & $\begin{array}{c}\text { Modalidad de enseñanza: } \\
\text { presencial, semipresencial, } \\
\text { virtual }\end{array}$ \\
\hline A Coruña & 2009-2010 & 240 & Presencial \\
\hline Barcelona & 2009-2010 & 240 & Presencial y semipresencial \\
\hline Carlos III de Madrid & $\begin{array}{l}\text { 2009-2010 } \\
\text { (fin de este grado en 2016-2017) }\end{array}$ & 240 & Presencial y semipresencial \\
\hline Complutense de Madrid & $2009-2010$ & 240 & Presencial \\
\hline Extremadura & 2011-2012 & 240 & Presencial y semipresencial \\
\hline Granada & 2010-2011 & 240 & Presencial \\
\hline León & 2010-2011 & 240 & Presencial \\
\hline Murcia & 2010-2011 & 240 & Presencial \\
\hline Salamanca & $2008-2009$ & 240 & Presencial \\
\hline Valencia Estudi General & $2009-2010$ & 240 & Presencial \\
\hline Zaragoza & $2008-2009$ & 240 & Presencial \\
\hline Universitat Oberta de Catalunya & $2009-2010$ & 240 & Virtual \\
\hline
\end{tabular}

Fuente: Elaborado por los autores (2016).

Para el diseño del título de Grado en Información y Documentación responsables de los centros y departamentos que impartían los títulos de Diplomado y Licenciado en Documentación se reunieron desde 2003 para planificar el futuro título. Como consecuencia de estas reuniones se redactó el Libro Blanco del Título de Grado en Información y Documentación (Agencia Nacional de Evaluación de la Calidad y Acreditación, 2004) donde se recogían las competencias genéricas y específicas que debían de tener los futuros titulados. Respecto a las orientaciones profesionales se proponían las salidas profesionales que ya tenían los diplomados y licenciados en Documentación, y específicamente en los siguientes tipos de centros:

1) Bibliotecas generales, ya sean nacionales, públicas, universitarias, de centros de enseñanza primaria y secundaria, parlamentarias etc.

2) Bibliotecas especializadas y centros de documentación de la Administración Pública, de empresas, de medios de comunicación, editoriales y librerías, asesorías y consultorías legales, de informática y tecnologías, de servicios bibliográficos y documentales etc.

3) Archivos nacionales, archivos de instituciones y organismos de la Administración pública y archivos privados.

4) Empresas de creación y difusión de bases de datos, de creación de sistemas de almacenaje y recuperación de la información, sistemas de información, portales de Internet, empresas de creación de contenidos editoriales etc.

El proceso de transformación ve desaparecer el término Biblioteconomía presente en la titulación de primer ciclo previa al proceso del EEES - Diplomatura en Biblioteconomía y Documentación- pero que no ha sido ajeno a la pérdida de peso de este término en otros países, o en la investigación en Ciencias de la Información (Sugimoto et al. 2011). Tampoco hay que olvidar que ya en el proceso de aprobación del Título de Diplomado en Biblioteconomía y Documentación tras la reforma de 1987 se intentó que el título se llamase Diplomado en Ciencias de la Documentación, aspecto que generó cierto rechazo en varias asociaciones profesionales que consideraron que los estudios dejaban de lado a los bibliotecarios y las bibliotecas (Abadal, 1993). 
En 2005 se publica el Real Decreto que establece la configuración de los estudios de Grado (España, 2005) que es modificado por el Real Decreto 1393/2007, de 29 de octubre, por el que se establece la ordenación de las enseñanzas universitarias oficiales (España, 2007). Estas enseñanzas de Grado tienen como finalidad la obtención por parte del estudiante de una formación general, en una o varias disciplinas, con planes de estudio de 240 créditos, de formación teórica y práctica, lo que equivale a cuatro cursos académicos. La normativa establece que todos los Grados se clasifiquen dentro de alguna de las siguientes ramas del conocimiento: (a) Artes y Humanidades, (b) Ciencias, (c) Ciencias de la Salud, (d) Ciencias Sociales y Jurídicas, (e) Ingeniería y Arquitectura.

Los 240 créditos debían distribuirse entre lo siguiente: aspectos básicos de la rama de conocimiento, materias obligatorias u optativas, seminarios, prácticas externas, trabajos dirigidos, trabajo de fin de Grado u otras actividades formativas. La polémica en los estudios de Información y Documentación surgió en el diseño curricular, ya que la adscripción a la rama de conocimiento de Ciencias Sociales y Jurídicas obligaba a la impartición de 60 créditos de la misma, pero el Real Decreto 1393/2007 (España, 2007) no contempló ninguna materia de Documentación, y sí de otras doce materias: Antropología, Ciencia Política, Comunicación, Derecho, Economía, Educación, Empresa, Estadística, Geografía, Historia, Psicología y Sociología. Así, una parte del Plan de estudios del Grado de Información y Documentación se conforma obligatoriamente por materias ajenas a la Documentación. Si bien los conocimientos adquiridos por estas materias complementarias, pueden dar una formación más sólida a un titulado en Información y Documentación (pensemos en las asignaturas de Gestión adscritas a Empresa, la Estadística, el Derecho o la Historia), en la práctica se impide que la Documentación como asignatura pueda impartirse en ningún Grado de la rama de Ciencias Sociales.

Esta pérdida de peso real de la Documentación en el sistema universitario español no fue más que la continuación de la merma de la importancia de la disciplina de Documentación en la sociedad, la empresa y la política españolas.

La adaptación de los estudios de Documentación al Grado del EEES dio lugar a que algunas universidades españolas, además de implantar el Grado presencial, ofertaran estas enseñanzas en otras modalidades a distancia u online, y en la modalidad semipresencial, que combina la enseñanza en modo presencial y virtual en un entorno tecnológico. Aunque algunas ya tenían esa experiencia en las antiguas Diplomaturas de Biblioteconomía usando el software Moodle (Reyes; Sánchez, 2009).

Los títulos diseñados por la universidades, deben ser verificados por la el Consejo de Universidades y evaluados cada varios años por la Agencia Nacional de Evaluación de la Calidad y Acreditación (Aneca) que podrá dar una propuesta favorable o desfavorable para la continuidad de la impartición del Título en la Universidad.

Se constituye así un modelo que viene a denominarse 4+1, es decir, cuatro cursos para los títulos de Grado más un curso suplementario de Máster, para aquellos alumnos que deseen una especialización. En 2015 a través del Real Decreto 53/2015 (España, 2015) se abre la posibilidad a reducir los títulos de Grado a 180 créditos, es decir tres cursos académicos, y, por tanto a ampliar los Máster a dos, con un modelo denominado 3+2, aunque de escasa incidencia en 2017, y con fuerte resistencia por parte del mundo universitario, tal es así que como se señala en el Cuadro 2, el Grado en Información y Documentación de la Universidad Carlos III de Madrid, deja de ofertarse en el curso 2017-2018 para ser sustituido por un nuevo título: Grado en Gestión de la Información y Contenidos Digitales, también de 240 créditos, y por tanto de cuatro cursos lectivos.

Las universidades que imparten los Grados en Información y Documentación (INDO) han creado tablas de equivalencia entre las asignaturas que estaban presentes en las antiguas Diplomaturas en ByD y los nuevos Grados, estableciendo, de esta manera, un método claro para la convalidación de asignaturas en los Grados de INDO. Sin embargo para favorecer que los titulados con diplomaturas en ByD puedan cursar el Grado en INDO algunas universidades han creado cursos de adaptación de varias asignaturas, que permiten a los diplomados, una vez superadas las mismas, obtener el título de Grado en Información y Documentación. Varias universidades han 
implantado estos cursos, con mayoría de oferta en modalidad semipresencial, aunque algunas universidades lo imparten en modalidad presencial.

También varias universidades han optado por una estrategia tendiente a ofertar una doble titulación o doble Grado en Información y Documentación con otro título, con una mezcla de asignaturas de ambas titulaciones. De las doce universidades que han impartido el Grado en Información y Documentación, hasta el curso 2016-2017, cuatro universidades han ofertado titulaciones conjuntas del Grado INDO con otros títulos. Así las universidades de Barcelona y Extremadura lo han hecho con Comunicación Audiovisual, la Universidad de Murcia con Periodismo, y la Universidad de Salamanca, la más activa en este sentido, ha diseñado planes conjuntos de INDO con los títulos de Ciencia Política y de la Administración, Educación Social, Pedagogía e Ingeniería Informática de Sistema de Información. Todos estos dobles títulos tienen entre 248 y 378 créditos lo que los convierte en títulos de cinco cursos lectivos.

\section{Crisis de los estudios: ¿Falta de adecuación a la realidad?}

Se ha escrito mucho en España sobre esta pérdida de peso de los estudios de Documentación que se achaca a la escasa visibilidad social y profesional de las titulaciones, a la falta de adaptación a la nueva realidad que han traído las tecnologías de la información y las comunicaciones, o al excesivo peso que sensibilidades más tradicionales de estos estudios han seguido ejerciendo. La evolución del alumnado matriculado en las antiguas Diplomaturas y Licenciaturas en Documentación es un claro ejemplo de la pérdida de importancia de estos estudios universitarios.

La Figura 1 presenta la evolución del número de alumnos matriculados tanto en la Diplomatura de Biblioteconomía en España, como en la Licenciatura en Documentación. Como puede comprobarse, el punto de

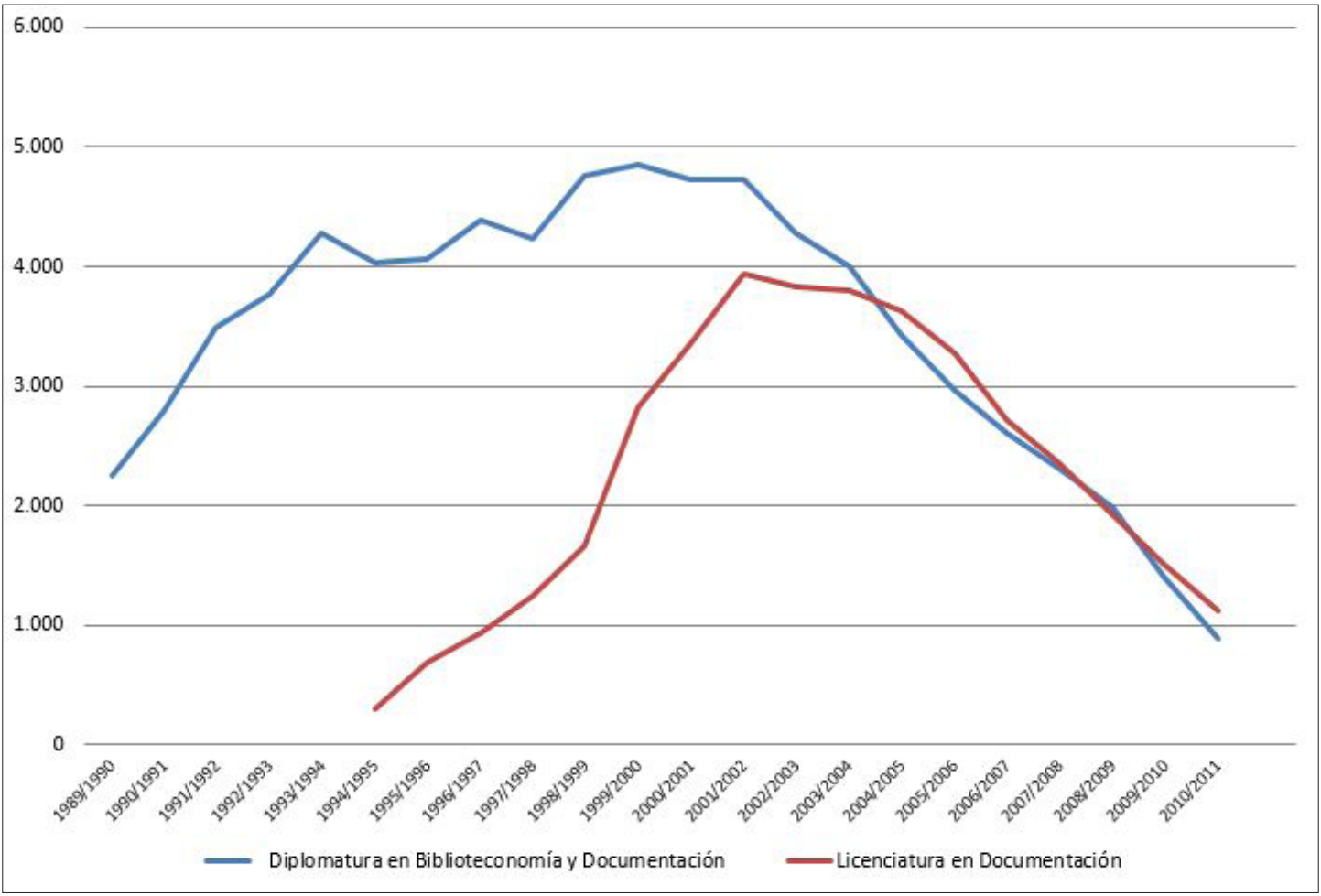

Figura 1. Evolución de los alumnos matriculados en la Diplomaturas y Licenciatura de Documentación en España. (Del curso 1988/1989 al curso 2010/2011).

Fuente: Instituto Nacional de Estadística (2016) <http:/ http://www.ine.es/>. 
inflexión en la Diplomatura lo supuso el curso 2000/2001 con una caída progresiva de las inscripciones que ya no se recuperaría. Mientras en la Licenciatura en Documentación esta caída comenzaría en el curso 2001/2002.

Este fenómeno que algunos han dado en Ilamar la“crisis de los estudios de Documentación en España"y que ha tenido una clara visibilidad en ese descenso paulatino del número de alumnos matriculados en estas titulaciones, ha generado arduos debates desde el interior de los estudios de Documentación, y que comienza a exteriorizarse en diversos foros y artículos sobre todo a partir de 2000, cuando se comienza a observar en la universidad española la pérdida alarmante de alumnos en las antiguas diplomaturas y licenciaturas de Documentación señaladas, y que se acentuará en el periodo de adecuación de los estudios de Documentación al EEES. De hecho en los nuevos Grados en Información y Documentación la escasez de alumnado sigue siendo una constante (Ortiz-Repiso; Calzada-Prado; Aportela-Rodríguez, 2013; Ortiz-Repiso, 2015).

En los debates señalados se puso de manifiesto la caída del número de alumnos matriculados en estas enseñanzas universitarias en España, la escasa adecuación del mundo académico al mundo profesional, también la existencia de otros problemas de fondo como la automatización creciente frente a una enseñanza centrada en los procedimientos artesanales; o la permanente crisis de identidad de la Ciencia de la Información - debido a su pluralidad paradigmática y metodológica de un campo interdisciplinario - (Chaín-Navarro; Muñoz-Cañavate, 2009). El Profesor Emilio Delgado puso de manifiesto en diversos trabajos este problema (Delgado, 2003, 2007, 2008) con una pérdida paulatina de alumnado que fue generando la desaparición de algunas diplomaturas, licenciaturas y doctorados. En su trabajo "El ocaso de las enseñanzas universitarias de Documentación en España" publicado primero en la lista de discusión Iwetel en 2007 y que generó un extenso debate, señalaba textualmente: "La conclusión no puede ser más desalentadora: mientras que la Universidad española crece y remonta la crisis producida en años anteriores como consecuencia del bajón demográfico, la ByD se hunde estrepitosamente" (Delgado, 2008, p.128). Causas analizadas por distintos autores que han buscado explicar las razones de este declive señalan algunas soluciones que permitirían crear un punto de inflexión al apuntar que la explosión de la información ha creado un nuevo espacio donde la información transdisciplinaria obliga tanto a académicos como a profesionales del área de Biblioteconomía y Documentación a resituarse y acomodarse en los próximos años (García-Marco, 2008a, 2008b, 2013; Ortiz-Repiso; Calzada-Prado; Aportela-Rodríguez, 2013; Ortiz-Repiso, 2015).

A la denominada crisis de los estudios de Documentación no ha sido ajena la tensión existente en la Biblioteconomía y la Documentación en España. Puede decirse que los orígenes de la Documentación española en el ámbito universitario crearon un perfil académico más orientado hacia salidas laborales clásicas como las enfocadas al mundo de las bibliotecas y los archivos. Así, en 2000 la profesora Purificación Moscoso apuntaba algunas razones entre las que destacaba el perfil humanístico de los docentes. Moscoso señalaba:

[...] para una gran parte de estos profesores la tecnología no tenía cabida alguna en sus tareas docentes e investigadoras" de manera que "el recelo hacia la gestión y uso de la información electrónica ha afectado de manera decisiva en el retraso con que el proceso de integración de tecnologías en las enseñanzas universitarias de Documentación ha tenido lugar en nuestras universidades (Moscoso, 2000, p.628).

García Marco describe muy bien como la llegada de la explosión digital a raíz de la explosión del World Wide Web en los años noventa con el despliegue de los estudios de Diplomado, Licenciado y Doctorado en Documentación daría lugar a una ruptura del profesorado entre los "modernos"y los "antiguos", algo que también ocurriría en el mundo de los profesionales y con un alumnado que veía unos planes de estudio obsoletos en un mundo extremadamente cambiante (García-Marco, 2013), y que se manifiesta en una permanente tensión entre la Librarianship y la Information Science (López-Borrull; Cobarsí-Morales, 2015). 
En la misma línea, el estudio de los centros de prácticas que se ofertan en la asignatura de prácticas curriculares del Grado en Información y Documentación de las distintas universidades nos da un perfil de la orientación profesional que se intenta conseguir con las mismas, y en cierto modo es un reflejo de las distintas sensibilidades del cuerpo docente que imparte las asignaturas en esas titulaciones. Los estudios realizados en 2015 sobre los centros donde realizan sus prácticas los alumnos de los Grados en Información y Documentación (Chaín-Navarro; Larios-Suárez; Muñoz-Cañavate, 2015) nos indican claramente que los centros varían escasamente respecto al estudio sobre el Prácticum de las antiguas, y desaparecidas, Diplomaturas de Biblioteconomía y Documentación en los que se demostraba la escasa incidencia de los nuevos nichos de mercado en la oferta de centros de prácticas de las universidades (Chaín-Navarro; Muñoz-Cañavate; Blanco-Faura, 2009b). Mayoritariamente los centros ofertados se encontraban en el ámbito de las bibliotecas, archivos y labores documentales tradicionales.

Esta situación también se ha debatido a nivel internacional. En 1995 Cronin insistía en lo que consideraba una tensión intolerable entre la Information Science y la Library Science, que aunque se habían integrado en un concepto más amplio como la Library and Information Science (LIS) sólo coexistían o cohabitaban sin ninguna integración (Cronin, 1995). Aunque Gorman nos decía en 1999 que el cambio fundamental en la educación lo ha supuesto la transformación de la biblioteconomía en ciencia de la información y más concretamente en gestión de la información, asegurando que los profesionales de la ciencia de la información debían ver el futuro de la profesión para introducir los cambios necesarios en los programas educativos (Gorman, 1999).

La escasa proyección profesional también ha tenido su reflejo en los puestos de trabajo ofertados por las Administraciones donde los títulos universitarios en Documentación no han tenido, en ocasiones, la debida consideración. Autores como José Antonio Moreiro analizan la situación de la profesión de bibliotecario-documentalista en España, estudiando la influencia de los planes de estudio en el mercado laboral, y entre las conclusiones destaca que la profesión y los estudios -después de más de treinta años- son poco conocidos por parte de la sociedad (Moreiro, 2014).

\section{Conclusión}

Los estudios de Grado en Información y Documentación sustituyen, junto con los Máster en Documentación, a las antiguas Diplomatura en Biblioteconomía y Documentación y Licenciatura en Documentación. Sin embargo, existe, tal y como se ha descrito, una corriente académica en España que ha puesto de manifiesto la escasa adaptación de los estudios de Información y Documentación a las nuevas realidades profesionales que han tenido como base a las tecnologías de la información y de las comunicaciones y, por tanto, la falta de adecuación de lo estudiado en los Grados universitarios al mercado laboral. De manera que nuevos puestos de trabajo relacionados con la gestión de la información se cubren con profesionales que proceden de cualquier titulación.

La situación descrita para España no es aislada en el mundo. Esa tensión señalada entre la Information Science y la Library of Science juega un papel relevante en la búsqueda de nuevas perspectivas profesionales para el mundo de la Biblioteconomía y Documentación en España. Y ha sido tratada en otros países. Jaye Weatherburn y Ross Harvey de la Universidad de Melbourne señalan que para evitar que los profesionales de la información se vuelvan obsoletos, en un paisaje de la información constantemente cambiante, necesitan abrir su mente a nuevos escenarios, adaptabilidad y un nuevo pensamiento conceptual, a la vez que educadores apasionados capaces de inspirar y crear un entorno desde el cual pueda surgir una nueva generación de profesionales de la información. De manera que a menos que se produzca esta nueva raza, los profesionales de la información se volverán irrelevantes en áreas donde deberían ser predominantes, fracasando en su misión de preservar el patrimonio cultural y proporcionar acceso universal a la información (Weatherburn; Harvey, 2016). Por su parte, Lawson, Krol y Kowatch, de la Universidad de Michigan, inciden en que aunque los nuevos perfiles que podrían incluirse en los estudios de Librarianship and Information Science darían un fuerte impulso a estos estudios estos nuevos perfiles no se encuentran en los asignaturas de los 
grados y postgrados en LIS, lo que implica que los estudiantes no salen con la preparación suficiente (Lawson; Kroll; Kowatch, 2010).

La realidad española ha quedado corroborada con el estudio realizado por la Universidad Complutense de Madrid en 2017 para adecuar sus estudios de Documentación al mercado laboral. En el mismo se señala que la mayor parte de las ofertas de trabajo procede del sector privado donde se solicitan documentalistas con altos conocimientos en tecnología e informática, o que los profesionales de la Documentación son demasiado obreros y poco ingenieros (Blazquez, 2017), lo que podría explicarse en parte por la ausencia de conocimientos en programación por parte de los estudiantes en Documentación, lo que convierte posteriormente al profesional en un mero usuario de las aplicaciones que hacen otros.

En 1998 Spencer Johnson escribió ¿Quién se ha llevado mi queso?, un relato breve que sirve para comprender la necesidad de adaptación al cambio en todos los ámbitos de la existencia (Johnson, 1998). El mundo académico de la Documentación no es ajeno a una nueva realidad, la de transformación digital, con cambios acelerados y abrumadores. Si no adapta sus planes de estudio y sus objetivos puede verse desplazado en muy pocos años.

\section{Colaboradores}

Los dos autores han contribuido a la planificación y diseño del artículo, el análisis de los datos y su redacción final.

\section{Referencias}

Abadal, E. La formación en Biblioteconomía y Documentación en España. Documentación de las Ciencias de la Información, v.16, p.9-46, 1993. Disponible en: <http://revistas.ucm.es/index. php/DCIN/article/viewFile/DCIN9393110009A/20105>. Acceso en: 30 oct. 2016.

Abadal, E.; Miralpeix, C. La enseñanza de la Biblioteconomía y la Documentación en la universidad española a finales de los noventa. BiD: Textos Universitaris de Biblioteconomia i Documentació, n.2, 1999. Disponible en: <http://bid.ub.edu/02abamir.htm>. Acceso en: 30 oct. 2016.

Agencia Nacional de Evaluación de la Calidad y Acreditación. Libro blanco del título de grado en Información y Documentación. Madrid: Aneca, 2004. Disponible en: <http://www.aneca.es/ var/media/150424/libroblanco_jun05_documentacion.pdf>. Acceso en: 1 mayo 2017.

Blázquez, M. La Documentación frente a las necesidades del mercado: el futuro de la carrera de Documentación en la Universidad Complutense de Madrid. Madrid: Universidad Complutense, 2017. Disponible en: <http://mblazquez.es/wp-content/uploads/ informe-documentacion112.pdf>. Acceso en: 1 mayo 2017.

Chaín-Navarro, C.; Larios-Suárez, V.; Muñoz-Cañavate, A. El Practicum en los Grados de Información y Documentación en España: un estudio sobre su adecuación al mercado laboral. In: Encuentro EDIBCIC, 7., 2015, Madrid. Actas... Madrid: Universidad Complutense, 2015. p.2-8. Disponible en: <http://eprints.ucm. es/34537/>. Acceso en: 16 feb. 2017.

Chaín-Navarro, C.; Muñoz-Cañavate, A. Information science courses and the graduate job context in Spain. Education for Information, v.27, n.2-3, p.115-126, 2009a. Available from: <http:// content.iospress.com/articles/education-for-information/ efi00876>. Cited: 10 Feb. 2017.

Chaín-Navarro, C:; Muñoz-Cañavate, A.; Blanco Faura, I. El Practicum en las Diplomaturas de Biblioteconomía y Documentación españolas. Revista General de Información y Documentación, v.19, n.1, p.145-160, 2009b. Disponible en: <http://revistas.ucm. es/index.php/RGID/article/view/RGID0909110145A>. Acceso en: 10 feb. 2017.

Chaín-Navarro, C.; Muñoz-Cañavate, A.; Salido Martínez, V. LIS education and web services in the public sector: The case of Spain'. Libri, v.58, n.4, p.246-256, 2008. Available from: <https:// www.degruyter.com/view/j/libr.2008.58.issue-4/libr.2008.025/ libr.2008.025.xml>. Cited: 10 Feb. 2017.

Cronin, B. Cutting the gordian knot. Information Processing and Management, v.31, n.6, p.897-902, 1995. Available from: <http:// www.sciencedirect.com/science/article/pii/030645739500017B >. Cited: 10 Jan. 2016.

Delgado, E. El ocaso de las enseñanzas universitarias de Documentación en España. Anuario ThikEPI, v.2, p.126-129, 2008. Disponible en: <https://dialnet.unirioja.es/descarga/ articulo/3190938.pdf>. Acceso en: 16 mayo 2016.

Delgado, E. Las cifras de la enseñanza universitaria en documentación en España: 2002. El Profesional de la Información, v.12, n.5, p.344-367, 2003. Disponible en: <http://eprints.rclis. org/12858/1/Las_cifras_de_la_documentacion_en_Espanna. pdf $>$. Acceso en: 10 mayo 2016.

Delgado, E. Las cifras de la infraestructura, profesión y educación documental en España: 2005. Anuario ThikEPI, v.1, p.207-217, 2007. Disponible en: <https://recyt.fecyt.es//index.php/ThinkEPI/ article/view/49190>. Acceso: 10 mayo 2016. 
España. Jefatura del Estado. Ley Orgánica 11/1983, de 25 de agosto, de Reforma Universitaria. Boletín Oficial del Estado, Madrid, 25 agosto. 1983. Disponible en: <https://www.boe.es/ buscar/doc.php?id=BOE-A-1983-23432>. Acceso en: 30 jul. 2016.

España. Ministerio de Educación y Ciencia. Acuerdo de 18 de diciembre de 1996, de Consejo de Universidades, por el que se formula a las Universidades recomendaciones, en relación con la organización de los planes de estudios conducentes a títulos universitarios oficiales. Boletín Oficial del Estado, Madrid, 18 dic. 1997. Disponible en: <http://www.boe.es/boe/dias/1997/01/17/ pdfs/A01895-01895.pdf>. Acceso en: 30 jul. 2016.

España. Ministerio de Educación y Ciencia. Real Decreto 1393/2007, de 29 de octubre, por el que se establece la ordenación de las enseñanzas universitarias oficiales. Boletín Oficial del Estado, Madrid, 29 oct. 2007. Disponible en: <http://www.boe.es/ buscar/doc.php?id=BOE-A-2007-18770>. Acceso en: 30 jul. 2016.

España. Ministerio de Educación y Ciencia. Real Decreto 1497/1987, de 27 de noviembre, por el que se establecen directrices generales comunes de los planes de estudio de los títulos universitarios de carácter oficial y validez en todo el territorio nacional. Boletín Oficial del Estado, Madrid, 27 nov. 1987. Disponible en: <https://www.boe.es/buscar/doc. php?id=BOE-A-1987-27707>. Acceso en: 30 jul. 2016.

España. Ministerio de Educación y Ciencia. Real Decreto 55/2005, de 21 de enero, por el que se establece la estructura de las enseñanzas universitarias y se regulan los estudios universitarios oficiales de Grado. Boletín Oficial del Estado, Madrid, 1 enero. 2005. Disponible en: <http://www.boe.es/diario_boe/txt. php?id=BOE-A-2005-1255>. Acceso en: 30 jul. 2016.

España. Ministerio de Educación y Ciencia. Real Decreto 912/1992, de 17 de julio, por el que se establece el título universitario oficial de Licenciado en Documentación y la aprobación de las directrices generales propias de los planes de estudios conducentes a la obtención de aquél. Boletín Oficial del Estado, Madrid, 17 jul. 1992. Disponible en: <https://www. boe.es/diario_boe/txt.php?id=BOE-A-1992-20359>. Acceso en: 30 jul. $201 \overline{6}$.

España. Ministerio de Educación, Cultura y Deporte. Real Decreto 43/2015, de 2 de febrero, por el que se modifica el Real Decreto 1393/2007, de 29 de octubre, por el que se establece la ordenación de las enseñanzas universitarias oficiales, y el Real Decreto 99/2011, de 28 de enero, por el que se regulan las enseñanzas oficiales de doctorado. Boletín Oficial del Estado, Madrid, 2 feb. 2015. Disponible en: <https://www.boe.es/boe/ dias/2015/02/03/pdfs/BOE-A-2015-943.pdf>. Acceso en: 30 oct. 2016.

España. Ministerio de Educación, Cultura y Deporte. Resolución de 10 de febrero de 2016, de la Dirección General de Política Universitaria, por la que se publica el Acuerdo del Consejo de Ministros de 22 de enero de 2016, por el que se determina el nivel de correspondencia al nivel del Marco Español de Cualificaciones para la Educación Superior del Título Universitario Oficial de Diplomado en Biblioteconomía y Documentación. Boletín Oficial del Estado, Madrid, 10 feb. 2016a. Disponible en: <https:// www.boe.es/boe/dias/2016/02/19/pdfs/BOE-A-2016-1692.pdf> Acceso en: 10 marzo 2017.

España. Ministerio de Educación, Cultura y Deporte. Resolución de 10 de febrero de 2016. De la Dirección General de Política Universitaria, por la que se publica el Acuerdo del Consejo de
Ministros de 22 de enero de 2016, por el que se determina el nivel de correspondencia al nivel del Marco Español de Cualificaciones para la Educación Superior del Título Universitario Oficial de Licenciado en Documentación. Boletín Oficial del Estado, Madrid, 10 feb. 2016b. Disponible en: <https://www. boe.es/boe/dias/2016/02/19/pdfs/BOE-A-2016-1699.pdf>. Acceso en: 10 marzo 2017.

Fuentes, M.E.; González, A. La investigación y la literatura especializada en Información y Documentación: una revisión. Scire: Representación y Organización del Conocimiento, v.7, n.2, p.11-38, 2001. Disponible en: <http://www.ibersid.eu/ojs/index. php/scire/article/view/1148>. Acceso en: 10 oct. 2016.

García-Marco, F.J. La Biblioteconomía y la Documentación española en la encrucijada. Ibersid, v.2, p.307-318, 2008a. Disponible en: <http://ibersid.eu/ojs/index.php/ibersid/article/view/2254> Acceso en: 30 nov. 2016

García-Marco, F.J. El movimiento ischools: posicionando los estudios de Biblioteconomía y Documentación en la Era de la Información. Anuario ThikEPI, v.3, p.95-99, 2008b. Disponible en: $<$ http://www.thinkepi.net/el-movimiento-ischools-posicionandolos-estudios-de-biblioteconomia-y-documentacion-en-la-erade-la-informacion>. Acceso en: 30 nov. 2016.

García-Marco, F.J. Educación y aprendizaje de la información y la documentación: raíces, desafíos y líneas de acción. El Profesional de la Información, v.22, n.6, p.489-504, 2013. Disponible en: <https://recyt.fecyt.es/index.php/EPI/article/view/epi.2013. nov.01>. Acceso en: 29 nov. 2016.

Gorman, G.E. The future for library science education. Libri, v.49, n.1, p.1-10, 1999.

Johnson, S. Who moved my cheese? New York: G.P. Putnam's Sons, 1998.

Lawson, J.; Kroll, J.; Kowatch, K. The new information professional: Your guide to careers in the digital age. New York: Neal-Schuman Publishers, 2010.

López-Borrull, A.; Cobarsí-Morales, J. ¿Tensión en el binomio LIS? Mensaje enviado a la lista de distribución de la Comunidad Académica Española. RedIRIS, 2015. Disponible en: <https:// listserv.rediris.es/cgi-bin/wa?A2=IWETEL;89cda88d.1502A>. Acceso en: 1 mayo 2017.

Mateu-lbars, J. Biblioteconomía en la universidad. Boletín de la Anabad, v.31, n.4, p.637-651, 1981.

Moneda-Corrochano, M. Las cifras de la enseñanza universitaria en documentación en España: 2012. Anuario ThinkEPI, v.8, p.24-38, 2014. Disponible en: <https://recyt.fecyt.es/index.php/ThinkEPI/ article/view/29548>. Acceso en: 29 oct. 2016.

Moreiro, J. A. Aún en torno a la formación universitaria, el empleo y la recesión. Anuario ThinkEPI, v.8, p.15-23, 2014. Disponible en: <https://recyt.fecyt.es//index.php/ThinkEPI/article/view/29560>. Acceso en: 29 mayo 2017

Moscoso, P. Tecnologías y Documentación: 25 años de desencuentros en la enseñanza de Documentación. In: Congreso Universitario de Ciencias de la Documentación, 1., 2000, Madrid. Teoría, historia y metodología de la Documentación en España (1975-2000). Madrid: Universidad Complutense, 2000. p.87-100.

Muñoz-Cañavate, A.; Larios-Suárez, V. Postgraduate studies in Library and Information Science in Spain. Education for 
Information, v.33, n.2, p.1-17, 2017. Available from: <http:// content.iospress.com/articles/education-for-information/ efi160087>. Cited: May 29, 2017.

Olvera-Lobo, M.D.; García-Caro, C. Presencia del área de Biblioteconomía y Documentación en otras titulaciones en las universidades españolas. In: Encuentro EDIBCIC, 5., 2000, Granada. Actas... Granada: Universidad de Granada, 2000, p.127-151.

Ortiz-Repiso, V. Repensar los estudios de Biblioteconomía y Documentación en España: traspasando los límites. BiD: Textos Universitaris de Biblioteconomia i Documentació, n. 35, 2015. Disponible en: <http://bid.ub.edu/es/35/ortiz.htm>. Acceso en: 29 nov. 2016.

Ortiz-Repiso, V.; Calzada-Prado, J.; Aportela-Rodríguez, I.M. ¿Qué está pasando con los estudios universitarios de Biblioteconomía y Documentación en España? El Profesional de la Información, v.22, n.6, p.505-514, 2013. Disponible en: <http:// www.elprofesionaldelainformacion.com/contenidos/2013/ noviembre/02.html>. Acceso en: 29 nov. 2016

Reyes, M.J.; Sánchez, C. La enseñanza semipresencial de Biblioteconomía y Documentación: una experiencia piloto en la Universidad de Extremadura (UEX). Boletín de la ANABAD, v.59, n.2, p.227-243, 2009.

Sanz-Casado, E. et al. Las disciplinas científicas como encrucijada de saberes: el caso de los estudios de Biblioteconomía y Documentación en las Universidades españolas. Revista General de Información y Documentación, v.11, n.1, p.167-181, 2001. Disponible en: <http://revistas.ucm.es/index.php/RGID/article/ viewFile/RGID0101120167A/10369>. Acceso en: 1 mayo 2016.

Sugimoto, C.R. et al. The shifting sands of disciplinary development: Analyzing north American library and information science dissertations using latent dirichlet allocation. Journal of the American Society for Information Science and Technology, v.62, n.1, p.185-204, 2011. Available from: <http://onlinelibrary.wiley. com/doi/10.1002/asi.21435/abstract>. Cited: Oct. 30, 2016.

Weatherburn, J., Harvey, R. Finding and forming the bold and the fearless: The future of LIS education in Australia. The Australian Library Journal, v.65, n.4, p.251-261. Available from: <http:// www.tandfonline.com/doi/full/10.1080/08164649.2016.1237 946>. Cited: May 1, 2017. 